\title{
Pregnant Women in Women-Only and Mixed-Gender Substance Abuse Treatment Programs: A Comparison of Client Characteristics and Program Services (DOI: 10.1007/s11414-006-9019-1)
}

\author{
Yih-Ing Hser, PhD
}

Noosha Niv, PhD

The last sentence of the Study Design section should read:

The other 7 CalTOP facilities admitted male clients only or did not have pregnant women clients and were excluded from the present analysis.

Address correspondence to Yih-Ing Hser, PhD, UCLA Integrated Substance Abuse Programs, Semel Institute for Neuroscience and Human Behavior, University of California, Los Angeles, CA 90025, USA. Phone:+1-310-4450874. E-mail:ysher@ucla.edu

Noosha Niv, PhD, UCLA Integrated Substance Abuse Programs, Semel Institute for Neuroscience and Human Behavior, University of California, Los Angeles, CA 90025, USA. Healthcare. 